\title{
Inhibition of osteoclast bone resorption activity through osteoprotegerin-induced damage of the sealing zone
}

\author{
RUILONG SONG ${ }^{*}$, JIANHONG GU* ${ }^{*}$ XUEZHONG LIU, JIAQIAO ZHU, QICHAO WANG, \\ QIAN GAO, JIAMING ZHANG, LAIYANG CHENG, XISHUAI TONG, \\ XINYI QI, YAN YUAN and ZONGPING LIU
}

College of Veterinary Medicine, Yangzhou University, and Jiangsu Co-innovation Center for Prevention and Control of Important Animal Infectious Diseases and Zoonoses, Yangzhou, Jiangsu 225009, P.R. China

Received March 20, 2014; Accepted July 2, 2014

DOI: $10.3892 /$ ijmm.2014.1846

\begin{abstract}
Bone remodeling is dependent on the dynamic equilibrium between osteoclast-mediated bone resorption and osteoblast-mediated osteogenesis. The sealing zone is an osteoclast-specific cytoskeletal structure, the integrity of which is critical for osteoclast-mediated bone resorption. To date, studies have focused mainly on the osteoprotegerin (OPG)-induced inhibition of osteoclast differentiation through the OPG/receptor activator of the nuclear factor kappa-B ligand (RANKL)/RANK system, which affects the bone resorption of osteoclasts. However, the effects of OPG on the sealing zone have not been reported to date. In this study, the formation of the sealing zone was observed by Hoffman modulation contrast (HMC) microscopy and confocal laser scanning microscopy. The effects of OPG on the existing sealing zone and osteoclastmediated bone resorption activity, as well as the regulatory role of genes involved in the formation of the sealing zone were examined by immunofluorescence staining, HMC microscopy, quantitative reverse transcription polymerase chain reaction (RT-qPCR), western blot analysis and scanning electron microscopy. The sealing zone was formed on day 5 , with beltlike protuberances at the cell edge and scattered distribution of cell nuclei, but no filopodia. The sealing zone was intact in the untreated control group. However, defects in the sealing zone were observed in the OPG-treated group $(20 \mathrm{ng} / \mathrm{ml})$ and the structure was absent in the groups treated with 40 and $80 \mathrm{ng} /$ $\mathrm{ml}$ OPG. The podosomes showed a scattered or clustered distribution between the basal surface of the osteoclasts and the well surface. Furthermore, resorption lacunae were not detected in the $20 \mathrm{ng} / \mathrm{ml}$ OPG-treated group, indicating the
\end{abstract}

Correspondence to: Professor Zongping Liu, College of Veterinary Medicine, Yangzhou University, 88 Daxuenanlu Road, Yangzhou, Jiangsu 225009, P.R. China

E-mail: liuzongping@yzu.edu.cn

*Contributed equally

Key words: osteoclast, osteoprotegerin, sealing zone, bone resorption, Rho GTPase, podosome loss of osteoclast-mediated bone resorption activity. Treatment with OPG resulted in a significant decrease in the expression of Arhgef8/Net1 and DOCK5 Rho guanine nucleotide exchange factors (RhoGEFs), 10 of 18 RhoGTPases (RhoA, RhoB, cdc42v1, cdc42v2, RhoU/Wrch1, RhoF/Rif, Rac2, RhoG, Rnd1 and RhoBTB1), ROCK1 and ROCK2. In conclusion, podosome distribution was affected by the OPG-induced inhibition of the expression of genes in the RhoGTPase signaling pathway. This resulted in damage to or destruction of the sealing zone, thus inhibiting osteoclast-mediated bone resorption activity.

\section{Introduction}

Osteoclasts have unique bone resorption activity. The dynamic equilibrium between osteoclast-mediated bone resorption and osteogenesis plays an important role in the bone remodeling process. Excessive bone resorption can cause a variety of diseases, such as osteoporosis, rheumatoid arthritis, multiple myeloma and periodontitis $(1,2)$. Therefore, investigation of bone absorption by osteoclasts is crucial for the development of effective therapeutic strategies.

The sealing zone is a cytoskeletal structure unique to osteoclasts that consists of a tight arrangement of the actin belt formed by podosomes $(3,4)$. The sealing zone provides a closed microenvironment for osteoclast-mediated bone resorption, thus, defining the area of bone resorption. Osteoclasts secrete protons and proteinases, which enter the microenvironment formed by the sealing zone to participate in the dissolution and absorption of the bone matrix and minerals (5-7). Furthermore, the sealing zone allows the tight adherence of osteoclasts to the surface of bone $(8,9)$.

The podosome, which is the structural unit of the sealing zone (6), is formed by the F-actin-binding core and the radiating actin fibers. During the osteoclast maturation process, the arrangement of podosomes changes from a spot-like to a clusterlike pattern that eventually transforms into a podosome belt or the sealing zone $(10,11)$. There is no essential difference between the podosome belt and the sealing zone, apart from the difference in density $(4,6)$. Myeloid cells, such as mononuclear macrophages and osteoclasts produce podosomes instead of the stress fibers and focal adhesions that are produced in other cells. Podosomes, focal adhesions and stress fibers share many common compo- 
nents $(5,12)$. The podosome has actin at its center, surrounded by scaffold proteins, kinases and integrins (6). Previous studies have indicated that the integrity of the sealing zone plays a decisive role in osteoclast-mediated bone resorption $(4,13)$.

RhoGTPases, which include at least 22 family members, affect the sealing zone by regulating the generation and arrangement of podosomes $(4,5,14-16)$. These enzymes are inactivated in the GDP-bound form and are activated in the GTP-bound form, playing an important role in cell signal transduction and the regulation of F-actin in the cytoskeleton $(15,16)$. Rho guanine nucleotide exchange factors (RhoGEFs) induced the upstream activation of RhoGTPases (17). Studies have shown that the expression of only Arhgef8/Net1 and DOCK5 among the RhoGEFs is altered during the formation and maturation of osteoclasts $(18,19)$; thus, it can be speculated that they play an important role in the regulation of the sealing zone. ROCK is the downstream effector of the Rho subfamily, the most extensively investigated member of this family, which includes ROCK1 and ROCK2 $(20,21)$.

Osteoprotegerin (OPG) markedly inhibits the transformation of osteoclast precursor cells into osteoclasts. Studies on the effects of OPG on bone resorption by osteoclasts have focused mainly on the OPG-induced inhibition of osteoclast differentiation through the OPG/receptor activator of the nuclear factor kappa-B ligand (RANKL)/RANK system (22-24). It has been reported that OPG promotes the separation of osteoclasts adhering to the bone matrix from the bone surface (25). However, the mechanisms involved have not yet been elucidated. In addition, to the best of our knowledge, the effects of OPG on the sealing zone and the underlying molecular mechanisms have not been reported to date.

In this study, the effects of OPG on the sealing zone of purified osteoclasts were observed. Furthermore, the expression of the RhoGTPase family members, Net1, DOCK5, ROCK (ROCK1 and ROCK2) was analyzed, in order to investigate the mechanisms responsible for OPG action on the sealing zone.

\section{Materials and methods}

Induction and purification of osteoclasts. Murine macrophagelike RAW264.7 cells were purchased from the Type Culture Collection of the Chinese Academy of Sciences, Shanghai, China. Studies have indicated that massive apoptosis of monocytes occurs during the generation of osteoclasts (26). According to this rule, the yield of osteoclasts can be increased by reducing the density of Raw264.7 cells (a mouse leukemic monocyte/macrophage cell line) at the early stage of inoculation and increasing the duration of induction. This will cause the greatest degree of apoptosis of undifferentiated monocytes. After the formation of a large number of osteoclasts, the monocytes can be removed by aspiration. This method was used for the generation of all osteoclasts used in this study.

For investigations of the formation of the sealing zone, RAW264.7 cells $\left(1562.5\right.$ cells $\left./ \mathrm{cm}^{2}\right)$ were incubated in $\alpha$-MEM culture medium containing $50 \mathrm{ng} / \mathrm{ml} \mathrm{M-CSF}$ and $60 \mathrm{ng} / \mathrm{ml}$ RANKL (PeproTech, Rocky Hill, NJ, USA), 10\% fetal bovine serum (FBS) (HyClone, Logan, UT, USA) and $100 \mathrm{U} / \mathrm{ml}$ penicillin/streptomycin (Sigma-Aldrich, St. Louis, MO, USA) for 1-5 days. Immunofluorescence (IF) staining (rhodamine phalloidin) were performed daily. IF staining was conducted to monitor the formation of the sealing zone. The time required for the formation of the sealing zone and the characteristics of the cells during the osteoclast differentiation process were observed using a Hoffman microscope (Leica, Wetzlar, Germany) on days 1-5.

For investigations of the effects of OPG (PeproTech) on the sealing zone, RAW264.7 cells $\left(1,562.5\right.$ cells $\left./ \mathrm{cm}^{2}\right)$ were incubated in $\alpha$-MEM culture medium containing $50 \mathrm{ng} / \mathrm{ml} \mathrm{M-CSF}$ and $60 \mathrm{ng} / \mathrm{ml}$ RANKL (PeproTech), 10\% FBS (HyClone) and $100 \mathrm{U} / \mathrm{ml}$ penicillin/streptomycin (Sigma-Aldrich). Based on the results of this staining protocol, osteoclasts with a visible sealing zone were incubated for $12 \mathrm{~h}$ with $\operatorname{OPG}(0,20,40$ and $80 \mathrm{ng} / \mathrm{ml}$ ). The changes of living osteoclasts in the sealing zone in these experiments were captured by Hoffman modulation contrast (HMC) microscopy. Moreover, IF staining (rhodamine phalloidin) was performed to monitor the effects of OPG (PeproTech) on the sealing zone.

IF staining. The cells obtained as described above were fixed for 15 min with 4\% paraformaldehyde (Sigma-Aldrich), and washed 3 times with phosphate-buffered saline (PBS). Subsequently, the cells were washed with $0.2 \%$ Triton X-100 (Amresco, Solon, $\mathrm{OH}$, USA) for $10 \mathrm{~min}$, then washed 3 times with PBS. The cells were sealed for $1 \mathrm{~h}$ with $500 \mu 12 \%$ bovine serum albumin (BSA; Sigma-Aldrich). Subsequently, the cells were stained for $1 \mathrm{~h}$ with rhodamine phalloidin (Invitrogen, Carlsbad, CA, USA) diluted in 2\% BSA and washed 3 times with PBS. During the analysis of the sealing zone formation, the cells were photographed immediately using an inverted fluorescence microscope (LSM510; Carl Zeiss, Jena, Germany). However, during the analysis of the effects of OPG on the sealing zone, the cells were stained for 15 min with 4',6-diamidino-2-phenylindole (DAPI), and washed 3 times with PBS. Finally, images were acquired using an inverted fluorescence microscope (Leica).

Quantitative reverse transcription polymerase chain reaction $(R T-q P C R)$. Total RNA was extracted from the cells treated with OPG using TRIzol reagent (Invitrogen) according to manufacturer's instructions. Reverse transcription was performed using the PrimeScript RT reagent kit with gDNA eraser (Takara Bio, Inc., Shiga, Japan). Subsequently, the mRNA expression levels of Arhgef8/Net1, DOCK5, the RhoGTPase family members, DOCK2 and other genes (MMP-9 and CAII) associated with bone resorption were detected with the 7500 Real-Time PCR System using SYBR-Green (Applied Biosystems, Carlsbad, CA, USA) following the manufacturer's instructions. The primers used in this study are presented in Table I.

Western blot analysis. Total proteins were extracted from the OPG-treated cells. The concentration of protein was determined using the BCA protein assay kit (Beyotime, Shanghai, China). Equal amounts of proteins were separated by sodium dodecyl sulfate-polyacrylamide gel electrophoresis [SDS-PAGE; $12 \%$ gel for glyceraldehyde 3-phosphate dehydrogenase (GAPDH) and $6 \%$ gel for ROCK1]. The proteins were then transferred onto nitrocellulose membranes and non-specific binding was blocked by incubation with $5 \%$ non-fat milk for $2 \mathrm{~h}$ at room temperature. Subsequently, the cells were incubated overnight at $4^{\circ} \mathrm{C}$ with primary anti-rabbit monoclonal antibodies (GAPDH and ROCK1) (Cell Signaling Technology, Beverly, MA, USA) diluted 
Table I. Primers used in real-time PCR.

\begin{tabular}{llll}
\hline Gene name & Accession no. & \multicolumn{1}{c}{ Upstream } & \multicolumn{1}{c}{ Downstream } \\
\hline RhoA & NM_016802 & CAAGGACCAGTTCCCAGAGG & CGCAGGCGGTCATAATCTTC \\
RhoB & NM_007483 & AAGTGGGTGCCCGAGGTA & CGAGGTAGTCATAGGCTTGGAT \\
RhoC & NM_007484 & GGAAGACCTGCCTCCTCATT & TCCACCTGCTTGCCATCCAC \\
Rac1 & NM_009007 & GCCTGCTCATCAGTTACACG & GACGCAATCTGTCATAATCTTC \\
Rac2 & NM_009008 & TGTGATGGTGGACAGTAAGCC & GAACCACTTGGCACGGACAT \\
Rac3 & NM_133223 & TGGTGAGCCCAGCCTCCTTT & CCGCAGCCGTTCAATCGTAT \\
RhoG & NM_019566 & CTGCTATACAACTAACGCCTTC & GTCCCACAGGTTTAGGTTCACG \\
Cdc42v1 & NM_009861 & CCTTTCTTGCTTGTTGGGAC & TGCGGCTCTTCTTCGGTT \\
Cdc42v2 & NM_001243769.1 & GTGGTCTCTCCATCCTCATT & GCCTCATCAAACACATTCTTC \\
RhoU (Wrch1) & NM_133955 & GAGAAGCCGGTGCCTGAAGA & GCTGGGAGTCTGAGTGCTGGAT \\
Rnd1 & NM_172612 & AGTGGTGGTCAGGTGTAAGC & GCACATAGGTCTCGGGATAG \\
Rnd2 & NM_009708 & GGTGCTGGTTGGCTGTAAG & CAGAAGATCGGGAGGAACA \\
RhoF (Rif) & NM_175092 & CCCGTCGGTGTTTGAGAAGTA & GAGGACGTTGTCGTAACTGGTG \\
RhoBTB1 & NM_001252638 & AAGGAGGAAGACCACTACCAG & GGACGAATGCCAGAAACAC \\
ROCK2 & NM_009072.2 & TTCACGTCCGACCTGTTAC & GGCACCTACGGCACTCTA \\
DOCK5 & XM_619261 & TGGACCTGCTAGGCTTACT & GATGTCTCCTATCAGCGAAA \\
Arhgef8/Net1 & NM_019671 & TGCCAGGCTTAAACGCTTGC & TGAATGCAGAAGGCGAACCG \\
CAII & K00811.1 & CATTACTGTCAGCAGCGAGCA & GACGCCAGTTGTCCACCATC \\
MMP-9 & NM_013599.3 & GCCCTGGAACTCACACGACA & TTGGAAACTCACACGCCAGAAG \\
GAPDH & GU214026 & ATGGTGAAGGTCGGTGTG & TGAAGGGGTCGTTGATGG \\
\hline
\end{tabular}

PCR, polymerase chain reaction; GAPDH, glyceraldehyde 3-phosphate dehydrogenase.

with 5\% BSA in TBST. The membranes were then washed 6 times in TBST piror to incubation with HRP-coupled goat antirabbit monoclonal antibody (Cell Signaling Technology) diluted with 5\% BSA in TBST. The membranes were then washed 6 times with TBST. The immune complexes were detected using the ECL system by standard scanning densitometry with the normalization of desitometry measures to GAPDH. The gray values of the blots were detected using Image Lab software.

Observation of bone resorption lacunae by scanning electron microscopy. Osteoclast purification and induction from the RAW264.7 cells was performed as described above, with the modification of cells being plated in 24-well plates containing bovine cortical bone slices. Following the formation of the sealing zone in osteoclasts, the cells were treated with $0 \mathrm{ng} / \mathrm{ml}$ and $20 \mathrm{ng} / \mathrm{ml} \mathrm{OPG}$ for $12 \mathrm{~h}$. Finally, the bone resorption lacunae of osteoclasts were observed by scanning electron microscopy.

Statistical analysis. In this study, each reaction was carried out in triplicate. The data of mRNA expression were analyzed by comparing their $2^{-\Delta \Delta \mathrm{Ct}}$ values. The results are presented statistically as the means \pm standard deviation (SD). Significance was assessed by one-way ANOVA. The results were compared between groups using ANOVA and the least significant difference (LSD) post-hoc test following the appropriate transformation to normalized data and equalized variance where necessary. Statistical analysis was performed using SAS 9.1.3 software (SAS Institute Inc., Cary, NC, USA); Values of $\mathrm{P}<0.05$ and $\mathrm{P}<0.01$ were considered to indicate statistically significant and highly significant differences, respectively.

\section{Results}

Differentiation and purification of osteoclasts. In order to reduce the effects of monocytes on the experimental results, the osteoclasts were purified at the stage where large quantities of osteoclasts were produced. When the sealing zone was formed, very few monocytes were found in the culture plate (Fig. 1A; HMC, day 5). Therefore, the presence of monocytes had little impact on the research results.

The characteristics of the osteoclasts were observed at the stage where the sealing zone was formed, which provided a reference for the addition of OPG. During the process of osteoclast differentiation, phalloidin-based IF staining was performed together with photographing live osteoclasts using an HMC microscope.

The sealing zone is composed of F-actin, to which phalloidin specifically binds. IF staining revealed that the formation of the sealing zone was mostly complete on day 5. HMC microscopy revealed that the cell morphology at this stage was characterized by a smooth cell edge, belt-like protuberances, no filopodia and a scattered arrangement of nuclei (Fig. 1A; HMC, day 5). In the subsequent experiments, OPG was added at the time at which the sealing zone had just appeared.

Effects of OPG on the sealing zone of osteoclasts. The evaluation of IF staining using an inverted fluorescence microscope revealed that, in the control group without the addition of OPG, the podosomes of the osteoclasts showed a strip-like, compact arrangement. On the inner side of the edge of cell, there were no podosomes; the sealing zone was intact (Fig. 1B, IF), and the 

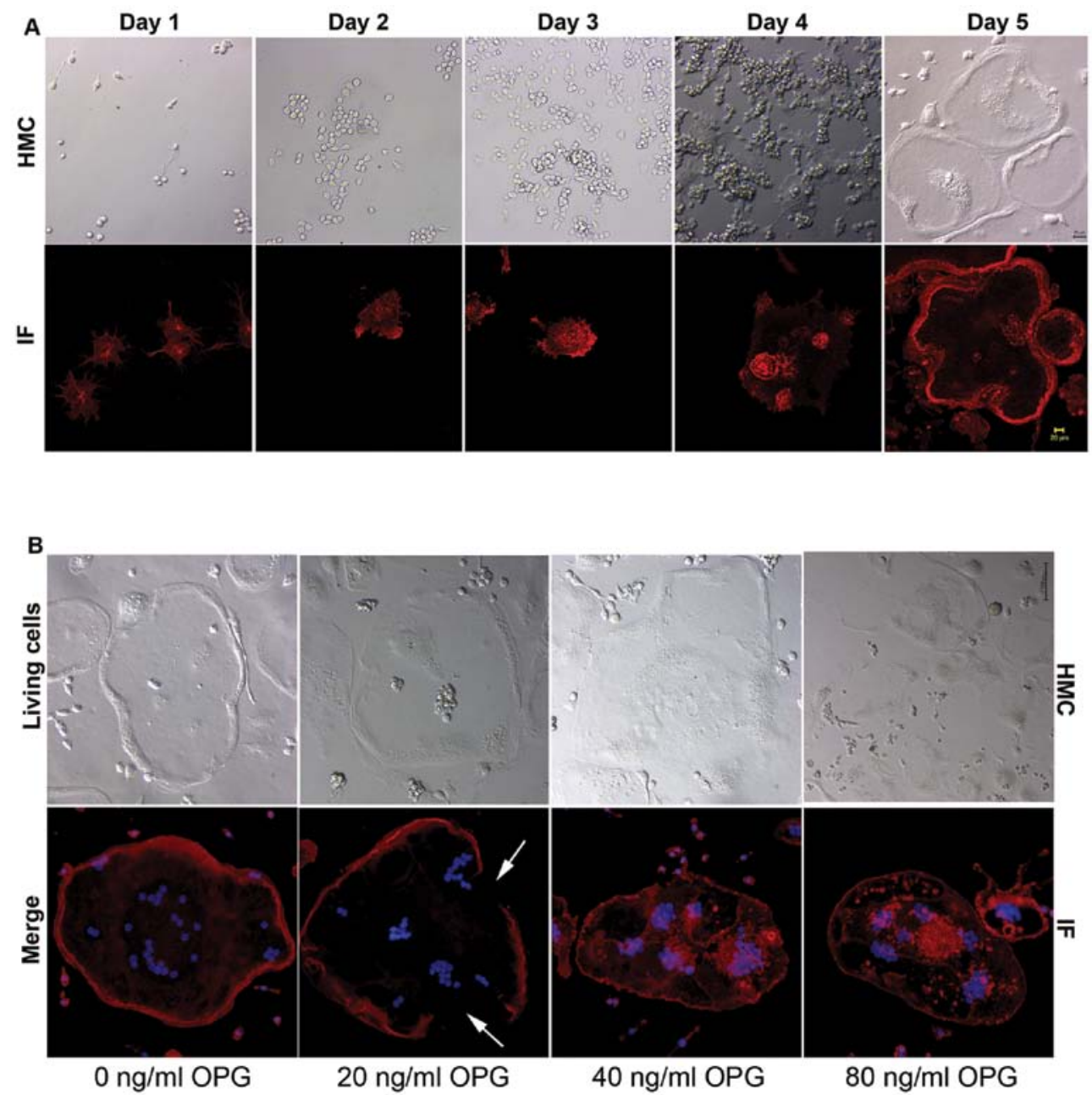

Figure 1. Formation process of the sealing zone and effects of osteoprotegerin (OPG) on the sealing zone of osteoclasts. (A) The formation process of the sealing zone was observed by Hoffman modulation contrast (HMC) microscopy and immunofluorescence (IF) staining (rhodamine phalloidin). The sealing zone was formed in day 5. (B) The effects of osteoprotegerin (OPG) on the sealing zone of osteoclasts by HMC and IF staining. The sealing zone of the osteoclasts showed defects in $20 \mathrm{ng} / \mathrm{ml}$ OPG-treated group (the white arrows), and completely disappeared in the $40 \mathrm{ng} / \mathrm{ml}$ and $80 \mathrm{ng} / \mathrm{ml}$ OPG-treated groups.

strip-like protuberances on the edge of the living cell were intact (Fig. 1B, HMC). By contrast, following treatment with $20 \mathrm{ng} /$ $\mathrm{ml} \mathrm{OPG}$, the sealing zone of the osteoclasts showed defects (Fig. 1B, IF). There were defects in the strip-like protuberances on the edge of the cell (Fig. 1B, HMC). It was surprising to note that the sealing zone of the osteoclasts in the $40 \mathrm{ng} / \mathrm{ml}$ and $80 \mathrm{ng} /$ $\mathrm{ml}$ OPG-treated groups had completely disappeared. Between the basal surface of the osteoclasts and the well surface, the podosomes showed a scattered or clustered distribution (Fig. 1B).

Expression of Arhgef8/Net1, DOCK5, RhoGTPase family members, DOCK and other genes associated with bone resorption. Although no significant differences in the expression of RhoC, Rac1, Rac3 and Rnd2 were observed following treatment with OPG at different concentrations, the mRNA expression levels of Arhgef8/Net1, DOCK5, other RhoGTPases and ROCK2 were all significantly downregulated (Fig. 2). Following treatment with OPG at 40 and $80 \mathrm{ng} / \mathrm{ml}$, the mRNA expression levels of MMP-9 and CAII were reduced to approximately $50 \%$ of the levels detected in the control group, while RhoU mRNA expression was reduced by approximately $80 \%$. Western blot analysis revealed that ROCK1 protein expression was sharply reduced following treatment with OPG in a concentration-dependent manner (Fig. 3).

Effects of OPG on resorption lacunae in osteoclasts. Following treatment with OPG, no visible resorption lacunae were observed in the bovine cortical bone slices (Fig. 4). However, in the control group not treated with OPG, many bead-like resorption lacunae were observed in the osteoclasts. These observations indicated the loss of osteoclast bone resorption function following treatment with OPG.

\section{Discussion}

In this study, the effects of OPG on the existing sealing zones of osteoclasts were investigated. Variations in the expression of genes (Arhgef8/Net1, DOCK5, RhoGTPase family members and ROCK) that play key regulatory roles in the formation of the sealing zone were determined before and after treatment with OPG. It was found that OPG treatment caused damage or complete destruction of the existing sealing zones of the osteoclasts. The osteoclasts lost the bone resorption function during this time. The expression of Arhgef8/Net1 and DOCK5 

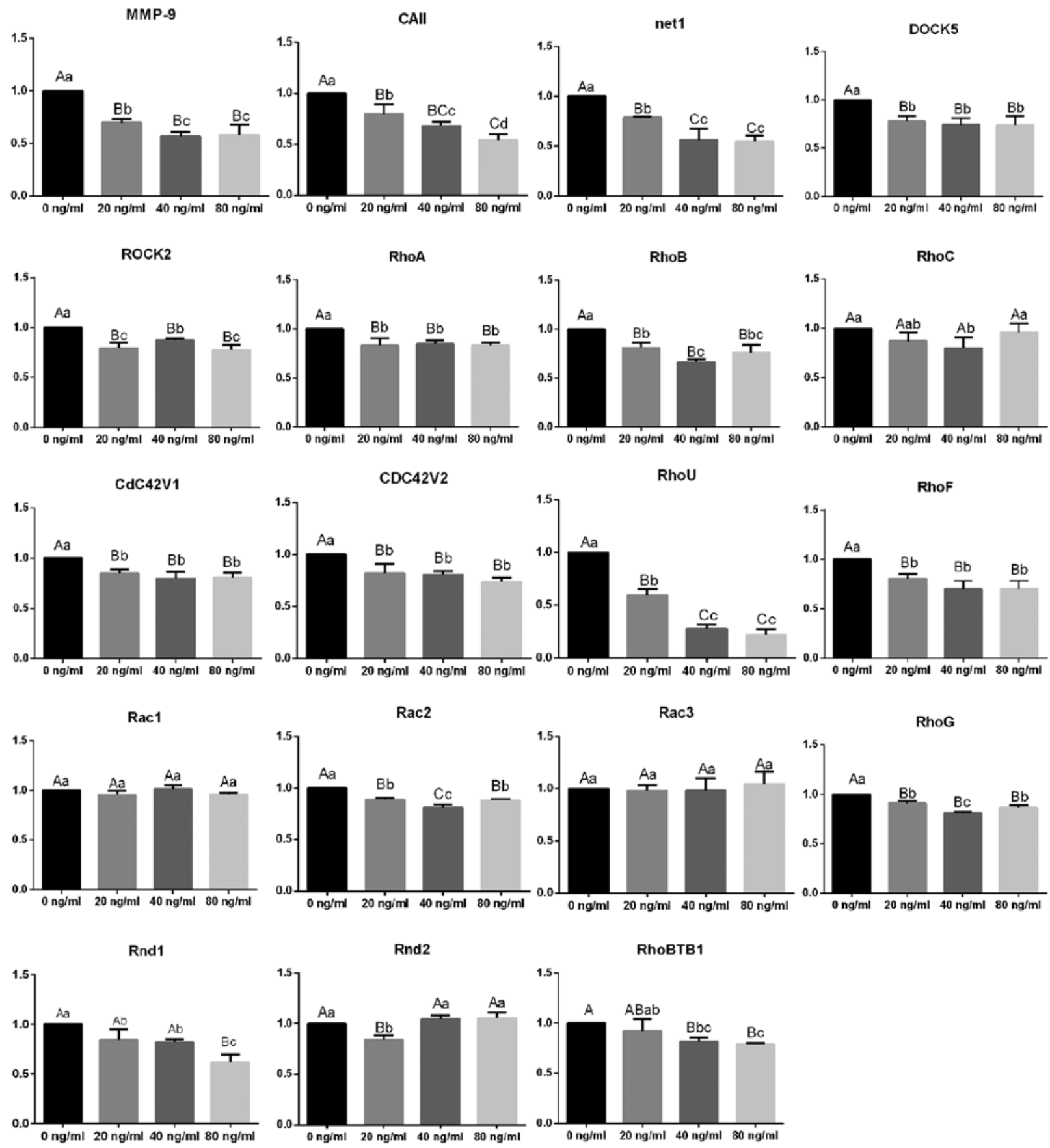

Figure 2. Expression of Arhgef8/Net1, DOCK5, RhoGTPase family, DOCK and other genes associatd with bone resorption. Histograms show the mRNA expression of bone resorption-related genes following osteoprotegerin (OPG) treatment analyzed by RT-qPCR. The abscissa represents the $0,20,40$ and $80 \mathrm{ng} /$ $\mathrm{ml}$ OPG treatment groups. The ordinate represents the relative mRNA expression. The same lower case alphabet letters and upper case alphabet letters indicate insignificant differences between the groups. Different lower case alphabet and upper case alphabet letters indicate significance $(\mathrm{P}<0.05)$ and high significance $(\mathrm{P}<0.01)$, respectively.

belonging to the RhoGEF family, 10 of 18 RhoGTPase family members, as well as 2 members of the ROCK family (ROCK1 and ROCK2) was downregulated significantly following treatment with OPG.

During the osteoclast differentiation process, IF staining revealed that the formation of the sealing zone was complete on day 5 (approximately). At this stage, HMC microscopy revealed that the cell morphology was characterized by a smooth cell edge, no filopodia, a scattered distribution of nuclei and striplike protuberances at the edge of cell. These characteristics were used as indicators for the addition of OPG in subsequent experiments to examine the effects of OPG on the existing sealing zone.

The sealing zone is a cytoskeletal structure unique to mature osteoclasts. It not only provides a favorable closed microenvironment for bone resorption, but also allows the firm adhesion of osteoclasts onto the bone surface. The integrity of the sealing zone is the prerequisite for the normal bone resorption function of osteoclasts $(4,13)$. In our study, in the control group not treated with OPG, the sealing zone remained intact. Following treatment with OPG at different concentrations, the sealing zones showed defects or had completely disappeared, and there was a scattered 

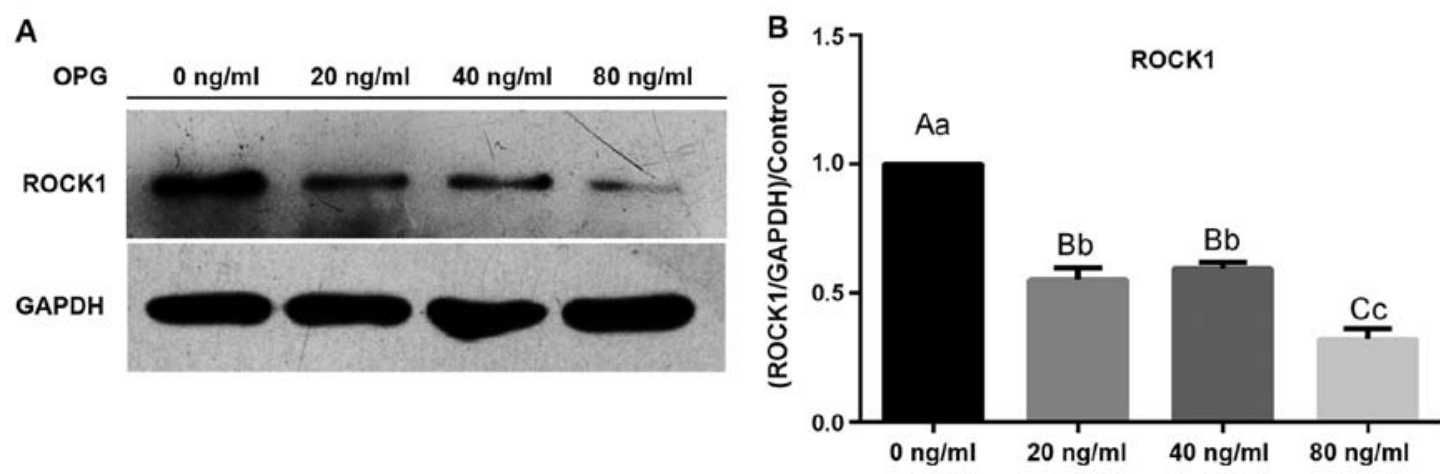

Figure 3. Protein expression of ROCK1. (A) Western blot analysis of ROCK1 protein expression in response to treatment with osteoprotegerin (OPG) at different concentrations. (B) Blots for ROCK1 were semi-quantified using Image Lab. The same lower case alphabet letters and upper case alphabet letters indicate insignificant differences between the groups. Different lower case alphabet letters and upper alphabet letters indicate significance $(\mathrm{P}<0.05)$ and high significance $(\mathrm{P}<0.01)$, respectively.
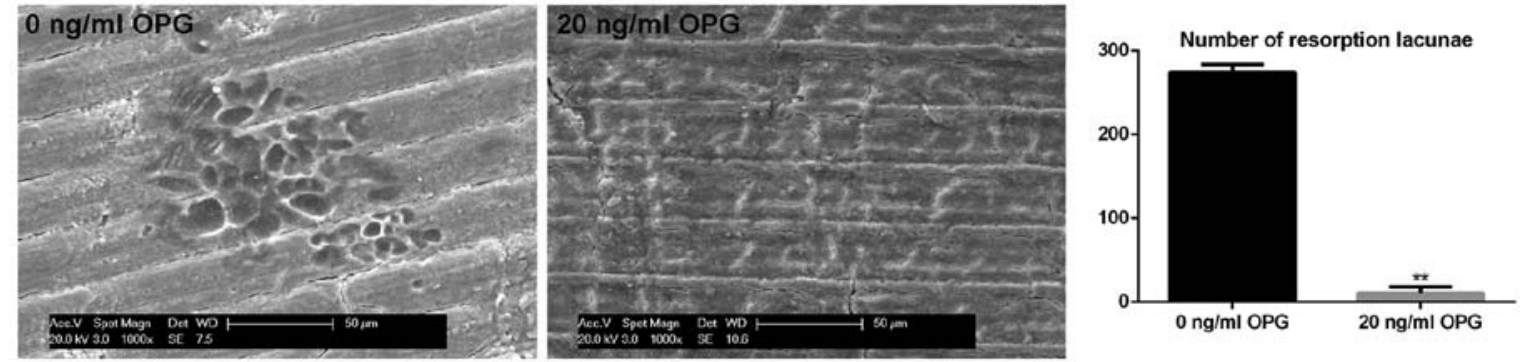

Figure 4. Effects of osteoprotegerin (OPG) on resorption lacunae in osteoclasts. (A) The resorption lacunae in osteoclasts from bovine bone were observed by scanning electron microscopy (magnification, $\mathrm{x} 1,000$ ). (B) We recorded the number of resorption lacunae formed on uniformly sized bovine bone slices in the $20 \mathrm{ng} / \mathrm{ml}$ OPG-treated group and control group in each test (the test was repeated 3 times).

or clustered arrangement of podosomes. In addition, investigations of the resorption lacunae showed that the osteoclasts had lost their bone resorption function in response to treatment with $20 \mathrm{ng} / \mathrm{ml}$ OPG. MMP-9 and CAII are genes involved in the process of osteoclast-mediated bone resorption. The proteins synthesized by the 2 genes are secreted into the sealing zone and participate in the decomposition of ossein and minerals $(27,28)$. The downregulation of MMP-9 and CAII mRNA expression corresponded to the effects of OPG on the bone resorption activity of osteoclasts. All these findings suggested that OPG caused the fracture or disappearance of the sealing zone by altering the arrangement of podosomes. Hence, the bone resorption activity of the osteoclasts was affected.

RhoGTPases are the molecular switches of signal transduction pathways, allowing the regulation of a variety of functions of the cell $(14,16)$. A number of studies have also confirmed that RhoGTPases control F-actin polymerization and adhesion, and play an important role in the formation of the sealing zone in osteoclasts (29-33). As shown by our previous study (34) and a study by other researchers (18), RhoH, RhoJ/TCL and RhoE/Rnd3 are not expressed during osteoclast differentiation and maturation. In addition, the expression of RhoQ/TC10, RhoV/Chp1 and RhoBTB2 is markedly inhibited during the formation of osteoclasts. It is thus indicated that RhoH, RhoJ/TCL and Rnd3, as well as RhoQ/TC10, RhoV/Chp1 and RhoBTB2 play no role in the sealing zone formation process. Moreover, the effect of the Miro family on actin is not known (15). The mRNA expression of the remaining 14 members of the RhoGTPase family was analyzed in osteoclasts following the disruption or disappearance of the sealing zone as a result of OPG treatment. No significant changes were detected in the mRNA expression of RhoC, Rac1, RhoE/Rnd3 and Rnd2 following treatment with OPG; however, a significant downregulation was observed in the mRNA expression of all the remaining 10 members (RhoA, RhoB, cdc42v1, cdc42v2, RhoU/Wrch1, RhoF/Rif, Rac2, RhoG, Rnd1 and RhoBTB1) of the RhoGTPase family ( $\mathrm{P}<0.01)$. In fact, RhoU expression was only approximately $20 \%$ of that observed in the control group following treatment with OPG. These results indicate that these 10 RhoGTPases play key a regulatory role in the OPG-induced defects or disappearance of the sealing zone in osteoclasts, with RhoU plays a more dominant role.

The upstream kinase, RhoGEF, activates RhoGTPases and catalyzes the conversion of GDP to GTP $(17,35)$. In our study, Arhgef8/Net1 and DOCK5 were significantly downregulated following treatment with OPG. In light of the interaction between RhoGEF and RhoGTPases and their roles in the formation of the sealing zone, we hypothesized that Arhgef8/Net1 and DOCK5 are involved in the regulation of RhoGTPases and are responsible for the damaging effects of OPG on the sealing zone.

As the major effector of the Rho GTPase family, ROCK receives the activating signal transmitted by RhoGTPases, which provide conditions that are critical for dynamic changes in the cytoskeleton, in particular, the nucleation and polymerization of actin $(36,37)$. The podosome contains an F-actin core, surrounded by scaffold proteins, kinases and integrins. The sealing zone is a large, compactly arranged F-actin ring composed of podosomes. Currently, there are no antibodies available for the detection of ROCK2; therefore, in our inves- 
tigations, we detected the mRNA expression levels of ROCK1 protein and ROCK2, both of which were significantly downregulated following treatment with OPG. These results indicate that ROCK1 and ROCK2 are involved in the OPG-induced arrangement of podosomes, which result in defects or destruction of the sealing zone. However, further studies are required to investigate the complex mechanism of OPG action on the sealing zone through the RhoGTPase signaling pathway.

In conclusion, OPG inhibited the expression of Arhgef8/Net1 and DOCK5 (RhoGEF) and 10 of the 18 members of the RhoGTPase family (RhoA, RhoB, cdc42v1, cdc42v2, RhoU/Wrch1, RhoF/Rif, Rac2, RhoG, Rnd1 and RhoBTB1), all of which are involved in the RhoGTPase signaling pathway. OPG also inhibited the 2 members of the ROCK family, ROCK1 and ROCK2. The OPG-mediated effects on the arrangement and formation of podosomes, resulted in defects or the destruction of the sealing zone and concomitant reduction in the bone resorption activity of the osteoclasts.

\section{Acknowledgements}

This study was supported by grants from the National Natural Science Foundation of China (nos. 31172373, 31302154 and 31372495), the Specialized Research Fund for the Doctoral Program of Higher Education (no. 20113250110003), the Priority Academic Program Development of Jiangsu Higher Education Institutions and the Graduate Innovation Project of Jiangsu Province (CXZZ12_0917).

\section{References}

1. Boyle WJ, Simonet WS and Lacey DL: Osteoclast differentiation and activation. Nature 423: 337-342, 2003.

2. Lane NE and Rehman Q: Osteoporosis in the rheumatic disease patient. Lupus 11: 675-679, 2002.

3. Zambonin-Zallone A, Teti A, Carano A and Marchisio PC: The distribution of podosomes in osteoclasts cultured on bone laminae: effect of retinol. J Bone Miner Res 3: 517-523, 1988.

4. Jurdic P, Saltel F, Chabadel A and Destaing O: Podosome and sealing zone: specificity of the osteoclast model. Eur J Cell Biol 85: 195-202, 2006

5. Ory S, Brazier H, Pawlak G and Blangy A: Rho GTPases in osteoclasts: orchestrators of podosome arrangement. Eur J Cell Biol 87: 469-477, 2008.

6. Luxenburg C, Geblinger D, Klein E, et al: The architecture of the adhesive apparatus of cultured osteoclasts: from podosome formation to sealing zone assembly. PLoS One 2: e179, 2007.

7. Nesbitt SA and Horton MA: Trafficking of matrix collagens through bone-resorbing osteoclasts. Science 276: 266-269, 1997.

8. Anderegg F, Geblinger D, Horvath P, et al: Substrate adhesion regulates sealing zone architecture and dynamics in cultured osteoclasts. PLoS One 6: e28583, 2011.

9. Saltel F, Destaing O, Bard F, Eichert D and Jurdic P: Apatitemediated actin dynamics in resorbing osteoclasts. Mol Biol Cell 15: 5231-5241, 2004

10. Luxenburg C, Parsons JT, Addadi L and Geiger B: Involvement of the Src-cortactin pathway in podosome formation and turnover during polarization of cultured osteoclasts. J Cell Sci 119: 4878-4888, 2006.

11. Akisaka T, Yoshida H and Suzuki R: The ruffled border and attachment regions of the apposing membrane of resorbing osteoclasts as visualized from the cytoplasmic face of the membrane. J Electron Microsc (Tokyo) 55: 53-61, 2006.

12. Schachtner H, Calaminus SD, Thomas SG and Machesky LM: Podosomes in adhesion, migration, mechanosensing and matrix remodeling. Cytoskeleton (Hoboken) 70: 572-589, 2013

13. Chiusaroli R, Knobler H, Luxenburg C, et al: Tyrosine phosphatase epsilon is a positive regulator of osteoclast function in vitro and in vivo. Mol Biol Cell 15: 234-244, 2004.
14. Itzstein C, Coxon FP and Rogers MJ: The regulation of osteoclast function and bone resorption by small GTPases. Small GTPases 2: 117-130, 2011

15. Vega FM and Ridley AJ: SnapShot: Rho family GTPases. Cell 129: $1430,2007$.

16. Coxon FP and Rogers MJ: The role of prenylated small GTP-binding proteins in the regulation of osteoclast function. Calcif Tissue Int 72: 80-84, 2003.

17. Rossman KL, Der CJ and Sondek J: GEF means go: turning on RHO GTPases with guanine nucleotide-exchange factors. Nat Rev Mol Cell Biol 6: 167-180, 2005.

18. Brazier H, Stephens S, Ory S, Fort P, Morrison N and Blangy A: Expression profile of RhoGTPases and RhoGEFs during RANKL-stimulated osteoclastogenesis: identification of essential genes in osteoclasts. J Bone Miner Res 21: 1387-1398, 2006.

19. Vives V, Laurin M, Cres G, et al: The Rac1 exchange factor Dock 5 is essential for bone resorption by osteoclasts. J Bone Miner Res 26: 1099-1110, 2011

20. Lee JH, Katakai T, Hara T, Gonda H, Sugai M and Shimizu A: Roles of p-ERM and Rho-ROCK signaling in lymphocyte polarity and uropod formation. J Cell Biol 167: 327-337, 2004.

21. Nakagawa O, Fujisawa K, Ishizaki T, Saito Y, Nakao K and Narumiya S: ROCK-I and ROCK-II, two isoforms of Rho-associated coiled-coil forming protein serine/threonine kinase in mice. FEBS Lett 392: 189-193, 1996.

22. Wang J, Chen TY, Qin S, Duan Y and Wang G: Inhibitory effect of metformin on bone metastasis of cancer via OPG/RANKL/ RANK system. Med Hypotheses 81: 805-806, 2013.

23. Hofbauer LC, Kühne CA and Viereck V: The OPG/RANKL/ RANK system in metabolic bone diseases. J Musculoskelet Neuronal Interact 4: 268-275, 2004

24. Shiotani A, Takami M,Itoh K, Shibasaki Y and Sasaki T: Regulation of osteoclast differentiation and function by receptor activator of NFkB ligand and osteoprotegerin. Anat Rec 268: 137-146, 2002.

25. O'Brien EA, Williams JH and Marshall MJ: Osteoprotegerin is produced when prostaglandin synthesis is inhibited causing osteoclasts to detach from the surface of mouse parietal bone and attach to the endocranial membrane. Bone 28: 208-214, 2001.

26. Song RL, Liu XZ, Zhu JQ, et al: RhoV mediated the apoptosis of RAW264.7 macrophages caused by osteoclast differentiation. Mol Med Rep (In press).

27. Borthwick KJ, Kandemir N, Topaloglu R, et al: A phenocopy of CAII deficiency: a novel genetic explanation for inherited infantile osteopetrosis with distal renal tubular acidosis. J Med Genet 40: 115-121, 2003.

28. Everts V,Delaissé JM, Korper W, Niehof A, Vaes G and Beertsen W: Degradation of collagen in the bone-resorbing compartment underlying the osteoclast involves both cysteine-proteinases and matrix metalloproteinases. J Cell Physiol 150: 221-231, 1992.

29. Dovas A, Gevrey JC, Grossi A, Park H, Abou-Kheir W and Cox D: Regulation of podosome dynamics by WASp phosphorylation: implication in matrix degradation and chemotaxis in macrophages. J Cell Sci 122: 3873-3882, 2009.

30. Gringel A, Walz D, Rosenberger G, et al: PAK4 and alphaPIX determine podosome size and number in macrophages through localized actin regulation. J Cell Physiol 209: 568-579, 2006.

31. Guegan F, Tatin F, Leste-Lasserre T, Drutel G, Genot E and Moreau V: p190B RhoGAP regulates endothelial-cell-associated proteolysis through MT1-MMP and MMP2. J Cell Sci 121: 2054-2061, 2008.

32. Osiak AE, Zenner G and Linder S: Subconfluent endothelial cells form podosomes downstream of cytokine and RhoGTPase signaling. Exp Cell Res 307: 342-353, 2005.

33. Tatin F, Grise F, Reuzeau E, Genot E and Moreau V: Sodium fluoride induces podosome formation in endothelial cells. Biol Cell 102: 489-498, 2010.

34. Song RL, Liu XZ, Zhu JQ, et al: New roles of filopodia and podosomes in the differentiation and fusion process of osteoclasts. Genet Mol Res 13: 4776-4787, 2014.

35. Schmidt A and Hall A: Guanine nucleotide exchange factors for Rho GTPases: turning on the switch. Genes Dev 16: 1587-1609, 2002.

36. Schofield AV and Bernard O: Rho-associated coiled-coil kinase (ROCK) signaling and disease. Crit Rev Biochem Mol Biol 48: 301-316, 2013.

37. Zeidan A, Paylor B, Steinhoff KJ, et al: Actin cytoskeleton dynamics promotes leptin-induced vascular smooth muscle hypertrophy via RhoA/ROCK- and phosphatidylinositol 3-kinase/ protein kinase B-dependent pathways. J Pharmacol Exp Ther 322: 1110-1116, 2007. 